\title{
Research and Development of Carbon Dioxide Refrigeration Technology
}

\author{
Wang $\mathrm{Qi}^{1}$, Li Guangpeng ${ }^{1,2^{*}}$ \\ ${ }^{1}$ School of Intelligent Manufacturing and Service, Shandong Institute of Commerce and Technology, Jinan, China \\ ${ }^{2}$ School of Mines, China University of Mining and Technology, Xuzhou, China
}

\begin{abstract}
With the agreement of the Kigali amendment to the Montreal Protocol, the timetable for the reduction of the mandate and the limitation of 18 controlled substances are being phased in. This has brought great challenges to the transformation, upgrading and sustainable development of China's refrigeration and air conditioning industry. It has brought great challenges to the transformation, upgrading and sustainable development of China's refrigeration and air conditioning industry. Carbon dioxide (CO2)is considered to be the most suitable and potential natural working medium due to its excellent environmental properties. This paper introduces the properties of $\mathrm{CO} 2$ refrigerant, $\mathrm{NH} 3 / \mathrm{CO} 2$ laminated refrigeration system, $\mathrm{CO} 2$ secondary refrigerant refrigeration system and $\mathrm{CO} 2$ trans-critical refrigeration cycle system, and analyzes three representative processes of $\mathrm{CO} 2$ refrigeration system.
\end{abstract}

\section{Introduction}

CFC refrigerants cause serious environmental damage, including damage to the atmospheric ozone layer and the greenhouse effect[1]. International consensus has been reached to completely ban $\mathrm{CFC}$ refrigerants and gradually restrict the use of $\mathrm{HCFC}$ refrigerants. Developed countries will completely phase out HCFC refrigerants by 2020 , and developing countries will cut their use to $35 \%$ by 2020 and completely eliminate them by $2030[2]$. As a natural refrigerant, the Ozone Depletion Potential value of CO2 is zero, and the Global Warming Potential value is one[3]. It has good environmental performance and can reduce the damage to the ozone layer. It is an ideal substitute refrigerant for $\mathrm{CFC}$. Therefore, $\mathrm{CO} 2$ refrigeration technology has broad prospects for development. This paper mainly introduces $\mathrm{NH} 3 / \mathrm{CO} 2$ cascade refrigeration system, $\mathrm{CO} 2$ secondary refrigerant refrigeration system and $\mathrm{CO} 2$ trans-critical refrigeration system commonly used in refrigeration and refrigeration industry.

\section{Properties of carbon dioxide}

Carbon dioxide has obviously characteristics, such as environmentally friendly, satisfactory thermal properties, good physical and chemical properties and wide sources. [4,5]First of all, it has a low boiling point and strong refrigeration capacity per unit volume. For a certain load, the volume flow through the system is small, thus facilitating the reduction of the size of the compressor. Secondly, carbon dioxide is non-toxic, non-flammable, non-explosive, and can be used with a variety of lubricating oils. It has no corrosion effect on alloy and other materials, and has good compatibility. Thirdly, the viscosity and density of $\mathrm{CO} 2$ are small, which can reduce the flow resistance and system diameter, further miniaturize the system, and save the room area. Most importantly, carbon dioxide is one of nature's constituents and has zero ozone destruction. However, the critical temperature of $\mathrm{CO} 2$ is $31^{\circ} \mathrm{C}$, making the high-pressure side pressure exceed the critical pressure under normal temperature cooling condition, up to $10 \mathrm{MPa}$.

\section{$3 \mathrm{NH} 3 / \mathrm{CO} 2$ cascade refrigeration system}

\subsection{The schematic diagram and $p-h$ diagram}

Figure 1 shows the schematic diagram of the compound refrigeration system. As shown in the figure, the system divides into two loops, the high-temperature circuit and the low-temperature circuit. NH3 environment-friendly refrigerant is used in the high-temperature circuit, and $\mathrm{CO} 2$, the natural working medium, is used in the lowtemperature circuit. The $\mathrm{CO} 2$ evaporator provides cooling for the refrigeration system, and the NH3 condenser exchanges heat with the outside world. The heat exchange temperature between the evaporator and the condenser depends on the ambient temperature and the system setting.

Figure 2 shows the p-h diagram of the cycle of the cascade refrigeration system. 4-1 is a low-temperature evaporation process for cooling, $1-2$ is the $\mathrm{CO} 2$ compression process, $2-3$ is the $\mathrm{CO} 2$ condensation process, the heat exchange with the 8-5 evaporation

* Corresponding author: 15098886718@163.com 
process in the NH3 system, and 3-4 is the throttling process. The NH3 system is similar to $\mathrm{CO} 2,6-7$ is the condensation process of heat exchange with the outside environment.

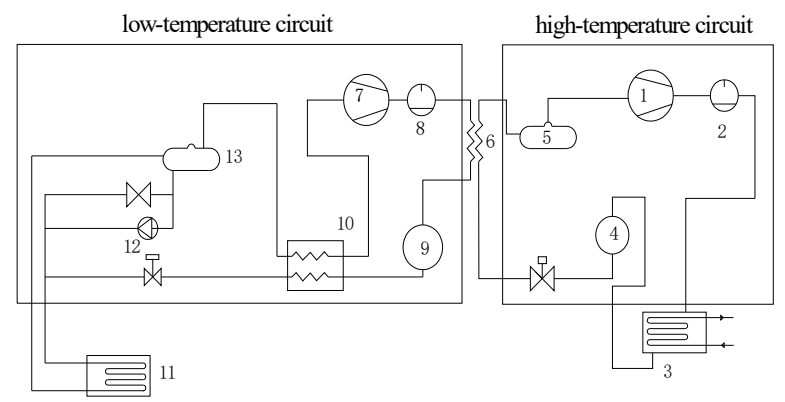

Figure 1. Schematic diagram of cascade refrigeration system 1- NH3 compressor. 2- oil gas separator. 3- NH3 condenser. 4$\mathrm{NH} 3$ accumulator. 5- $\mathrm{NH} 3$ gas liquid separator. 6- evaporative condenser. 7-CO2 compressor. 8- oil gas separator. 9-CO2 accumulator. 10- regenerator. 11- evaporator. $12-\mathrm{CO} 2$ pump. 13-CO2 gas liquid separator.

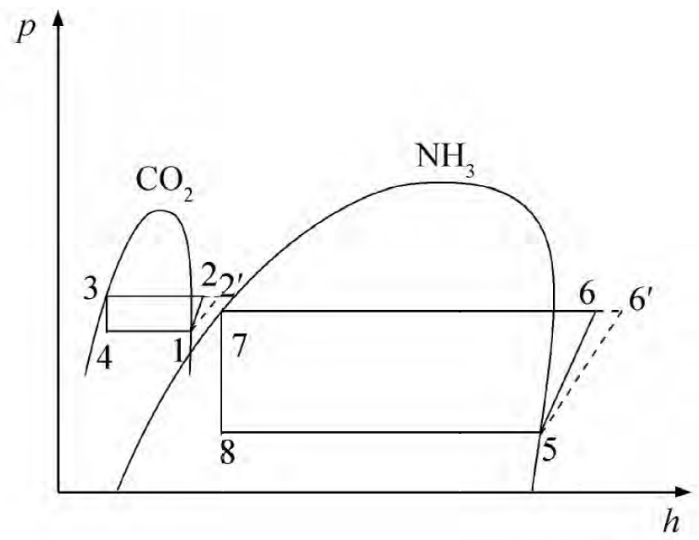

Figure 2. p-h diagram of cascade refrigeration system

\subsection{Application characteristics analysis}

$\mathrm{NH} 3 / \mathrm{CO} 2$ cascade refrigeration system can significantly reduce NH3 charging capacity, which is about $1 / 8$ of NH3 two-stage compression refrigeration system. Since the cryogenic refrigeration circulation system uses $\mathrm{CO} 2$ as the refrigerant, it can avoid the direct contact between NH3 and the cooled object, thus improving the safety of $\mathrm{NH} 3$ refrigeration circulation system. In the event of a major leakage event, $\mathrm{CO} 2$ will only form dry ice, which will not cause harm when it sublimates into gas, thus increasing the safety of system operation.

The design of the heat exchanger is a key equipment which affects the performance of the multilayer system.It involves two phase transition processes in which NH3 and $\mathrm{CO} 2$ occur simultaneously, which brings great challenges to the design of heat exchangers. In addition, the intermediate temperature of the heat exchanger directly affects the efficiency of the whole system. As for the selection of intermediate temperature, there are generally two ways: one is to maximize the refrigeration system, and the other is to make the pressure ratio of two compressors approximately equal. When the root data energy maximizes, the optimal intermediate temperature is determined as follows: at the same condensation temperature, there is an optimal intermediate temperature in the $\mathrm{NH} 3 / \mathrm{CO} 2$ overlapping cycle, and at this time, the COP under the corresponding working condition is maximum.In addition, COP changes very little within the $5 \mathrm{~K}$ temperature range of the optimal temperature. This also adds flexibility to the design and operation of the $\mathrm{NH} 3 / \mathrm{CO} 2$ complex system.For cold storages, especially those below $-40^{\circ} \mathrm{C}$, the efficiency of the overlapping system can be effectively improved after optimized design.

\section{$4 \quad \mathrm{CO} 2$ secondary refrigerant refrigeration system}

\subsection{The schematic diagram and $\mathrm{p}$-h diagram}

Figure 2 shows the schematic diagram of the $\mathrm{CO} 2$ refrigerant carrying refrigeration system. NH3 compressors can be single - or double - stage. Under the condition of low evaporation temperature, the temperature difference between evaporation and condensation of $\mathrm{NH} 3$ refrigeration system can be kept at a higher level through two-stage compression, while ensuring the efficiency of the compressor. As shown in the figure, high pressure and high temperature ammonia condenses into ammonia solution after heat exchange with the outside environment. The ammonia liquid flows through the throttle valve to the condensing evaporator for heat exchange with $\mathrm{CO} 2$, so as to liquefy the $\mathrm{CO} 2$ carrier coolant.

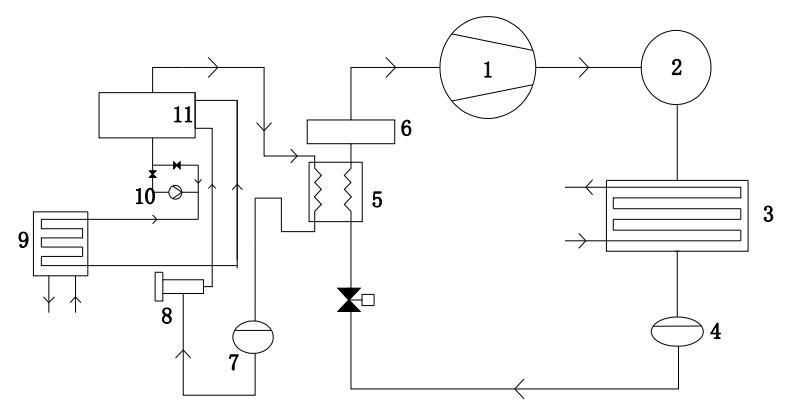

Figure 3. Schematic diagram of secondary refrigerant system 1- NH3 Single machine double stage compression. 2- oil gas separator. 3- NH3 condenser. 4- NH3 accumulator. 5evaporative condenser. 6- $\mathrm{NH} 3$ gas liquid separator. 7-CO2 accumulator. 8-Drying filter. 9- evaporator. 10- $\mathrm{CO} 2$ pump. 11$\mathrm{CO} 2$ gas liquid separator.

Driven by the pump, the $\mathrm{CO} 2$ refrigerant provides cooling capacity for the cold storage, and enters into the next cycle after gasification. It is worth mentioning that the optimal design of the heat exchanger is very important, because the $\mathrm{CO} 2$ refrigerating agent needs to exchange heat with $\mathrm{NH} 3$ to liquefy, and to provide cooling and gasification for the cold storage, in which the two-stage heat exchange temperature difference is needed to provide heat exchange. If the heat transfer temperature difference is large, a high COP of the system cannot be guaranteed. The $\mathrm{p}$-h diagram of refrigerant carrying refrigeration system is shown in Figure 4. 


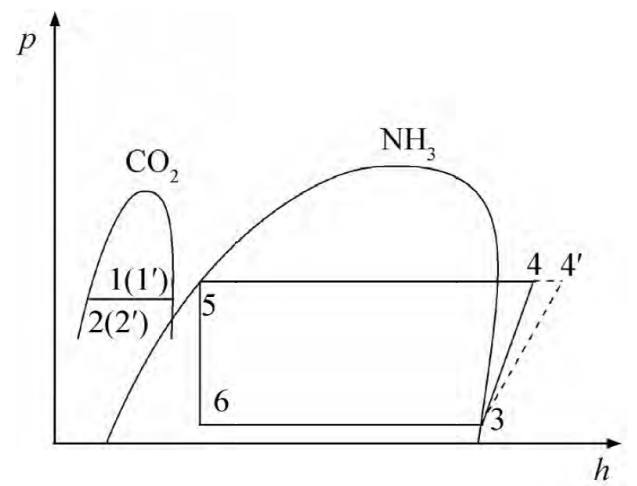

Figure 4. p-h diagram of secondary refrigerant system

\subsection{Application characteristics analysis}

The refrigeration system is generally used in occasions where the refrigeration temperature is $-50^{\circ} \mathrm{C} \sim 0^{\circ} \mathrm{C}$. The $\mathrm{CO} 2$ circulating side pressure is relatively high, especially in defrosting up to $4.0 \mathrm{Mpa} \sim 5.0 \mathrm{Mpa}$. Due to the environmental temperature, the pressure may be higher when the system is down.

As the $\mathrm{CO} 2$ circulating side is generally placed in dense areas such as cold storage and cold room, the pressure safety protection is particularly important. First of all, the pressure vessel and pipeline pressure capacity should be fully considered in the system design and installation process to ensure the pressure safety;Secondly, reliable pressure safety protection devices such as safety valve are set in the system to relieve pressure in time when the pressure exceeds the safe working pressure to ensure the safety of the system.Third, vent or recover part of the $\mathrm{CO} 2$ refrigerant during the shutdown to ensure that the $\mathrm{CO} 2$ circulating side pressure is in a safe range.

\section{CO2 trans-critical refrigeration system}

\subsection{The common $\mathrm{CO} 2$ refrigeration cycle}

Trans-critical cycle is the most common $\mathrm{CO} 2$ refrigeration cycle at present. The exothermic process of the cycle is above the critical point of $\mathrm{CO} 2$ and the endothermic process is below the critical point. In the trans-critical cycle, the heat absorption process of the cycle is carried out under subcritical conditions, and the heat transfer is completed by the latent heat. The cyclic cooling depends on sensible heat, which is completely different from subcritical condensation.

Figure 5 shows the principle of simple $\mathrm{CO} 2$ transcritical refrigeration system. 1-2 is the process of liquid $\mathrm{CO} 2$ gasification and endothermic refrigeration and the pressure in the evaporator is lower than the critical pressure of carbon dioxide. 2-3 is the process in which carbon dioxide gas is compressed in the compressor, and the pressure of carbon dioxide after compression is higher than the critical pressure. 3-4 is the process of constant pressure heat release of carbon dioxide gas in supercritical state.In this heat exchange process, carbon dioxide has no phase change and only transfers sensible heat. Therefore, the heat exchanger is called gas cooler rather than condenser. 4-1 is an adiabatic throttling process, and then constitutes a complete cycle.

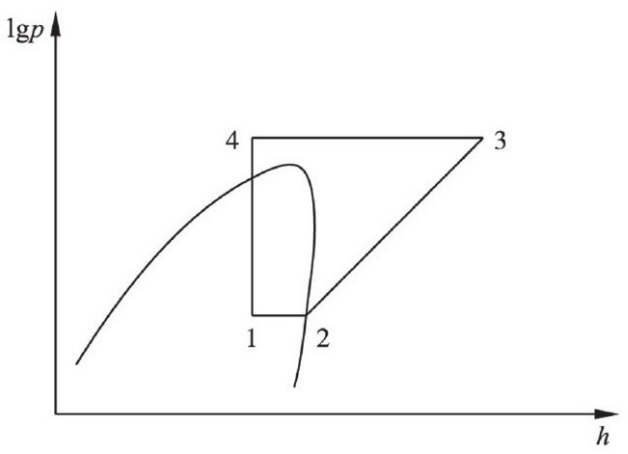

Figure 5. p-h diagram of trans-critical refrigeration system

\subsection{The principle of trans-critical refrigeration system with injector}

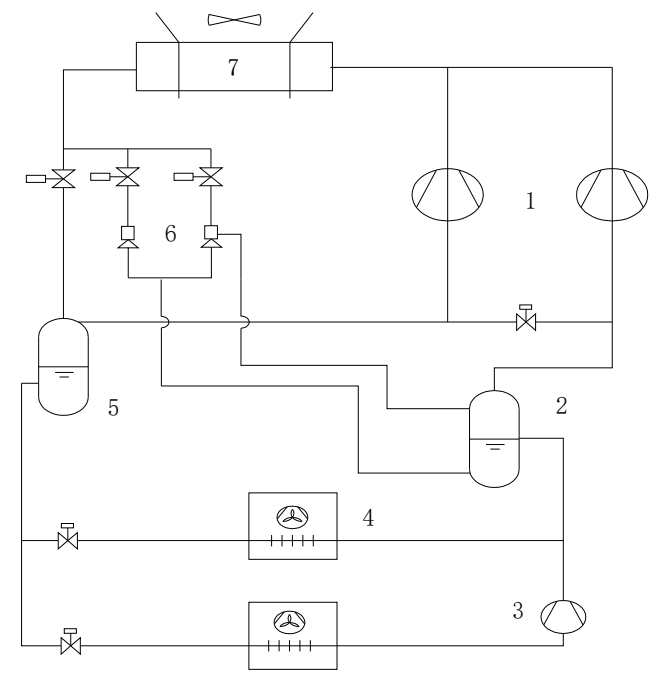

Figure 6. Schematic diagram of trans-critical refrigeration system

1- parallel units. 2- outlet reservoir. 3- maintenance unit. 4- aircooler. 5- Intermediate reservoir. 6-injector.7- evaporative condenser

Figure 6 shows the principle of trans-critical refrigeration system with injector. After using the injector, on the one hand, the compressor inlet pressure is increased, the compression ratio is reduced, and the compressor power consumption is reduced.On the other hand, it reduces the throttling loss when the fluid flows through the expansion valve. After flowing through the gas cooler, the supercritical carbon dioxide enters the main nozzle of the injector, and the flow passage area shrinks. According to the continuity equation, the fluid velocity increases and the pressure drops, which converts the fluid potential energy into kinetic energy. This causes the low-pressure carbon dioxide vapor in the evaporator to flow into the ejector nozzle. Subsequently, the workflow and the jetted jet are mixed in the mixing section. When they flow through the diffuser section, the area of the flow passage increases, the velocity of $\mathrm{CO} 2$ slows down, and the pressure increases, that is, the fluid kinetic energy is converted into potential energy.After the carbon dioxide from the ejector passes through the 
gas-liquid separator, part of it enters the compressor; The other part enters the evaporator after throttling.

\subsection{Application characteristics analysis}

$\mathrm{CO} 2$ compressor is a refrigeration equipment which makes $\mathrm{CO} 2$ pressurized and realizes conveying. The critical pressure of $\mathrm{CO} 2$ is high $(7.38 \mathrm{MPa})$ and the critical temperature is low $\left(31.3^{\circ} \mathrm{C}\right)$. Therefore, the operation of $\mathrm{CO} 2$ compressor requires high pressure resistance, higher requirements for pipes, fittings and valves, more accurate clearance control of moving parts, and the inlet temperature of the compressor is higher than the critical temperature. If there is water in the $\mathrm{CO} 2$ system, it will not only cause ice blocking, but also cause the corrosion of compressor due to the carbonic acid generated by the reaction between $\mathrm{CO} 2$ and water. Therefore, there are certain requirements on the materials of $\mathrm{CO} 2$ compressor. Because the internal pressure of $\mathrm{CO} 2$ compressor is higher, the requirement of oil return system inside the compressor is more strict.

\section{Conclusion}

As a refrigerant, $\mathrm{CO} 2$ meets the needs of environmentally friendly development, and can achieve energy conservation and reduce environmental damage. $\mathrm{NH} 3 / \mathrm{CO} 2$ cascade refrigeration system can meet the high requirements of safety and temperature in food freezing and refrigeration industry, and can be applied to large supermarkets, cold storage, quick-frozen tunnels and quick-frozen food, etc. The refrigeration system with $\mathrm{NH} 3$ as the refrigerant and $\mathrm{CO} 2$ as the refrigerant can greatly reduce the amount of ammonia filling, and ammonia is limited within the scope of the machine room and isolated from the dense working area such as the cold room, which greatly improves the safety of the system. $\mathrm{CO} 2$ trans-critical refrigeration system is developing rapidly in the field of food refrigeration and commercial supermarkets, and can generate high-quality heat sources through the recovery of high temperature condensing heat, so as to realize the safe, low-carbon and efficient utilization value.

\section{References}

1. Tian Yafen, Zhao Zhaorui, Xing Ziwen, Yang Yifan, Ma Jin, Yu Zhiqiang. Study on Application Scope for $\mathrm{CO} 2$ Cascade Refrigeration System and $\mathrm{CO} 2$ Secondary Refrigerant System[J]. Journal of Refrigeration, 2016,37(02),22-29

2. Liu Yefeng, Tang Danping, Meng Deren, Zhu Hongliang. Application Test of CO2 Two-stage Compression Refrigeration System in Refrigerator [J]. Fluid Machinery, 2019,47(08):76-80.

3. Yefeng Liu,Ying Sun,Dongliang Wang. Research on carbon dioxide transcritical refrigeration cycle with vortex tube[J]. IOP Conference Series: Earth and Environmental Science,2019,267(2).
4. Mikolaj Mastrowski,Jacek Smolka,Armin Hafner,Michal Haida,Michal Palacz,Krzysztof Banasiak. Experimental study of the heat transfer problem in expansion devices in CO 2 refrigeration systems[J]. Energy,2019,173.

5. Ma M,Yu J,Wang X. Performance evaluation and optimal configuration analysis of a $\mathrm{CO} 2 / \mathrm{NH} 3$ casc ade refrigeration system with faling film evaporatorcondenser[J]. Energy Conversion and Management,2014,79(2):224-231.3 